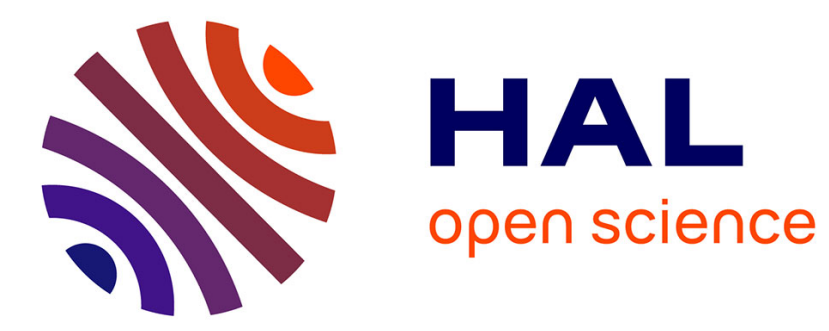

\title{
INTERFACE RECOMBINATION IN GaAs-GaAlAs QUANTUM WELLS
}

B. Sermage, M. M. Pereira, F. Alexandre, J. Beerens, R. Azoulay, C. Tallot, A. Jean-Louis, D. Meichenin

\section{> To cite this version:}

B. Sermage, M. M. Pereira, F. Alexandre, J. Beerens, R. Azoulay, et al.. INTERFACE RECOMBINATION IN GaAs-GaAlAs QUANTUM WELLS. Journal de Physique Colloques, 1987, 48 (C5), pp.C5-135-C5-138. 10.1051/jphyscol:1987525 • jpa-00226729

\section{HAL Id: jpa-00226729 https://hal.science/jpa-00226729}

Submitted on 1 Jan 1987

HAL is a multi-disciplinary open access archive for the deposit and dissemination of scientific research documents, whether they are published or not. The documents may come from teaching and research institutions in France or abroad, or from public or private research centers.
L'archive ouverte pluridisciplinaire HAL, est destinée au dépôt et à la diffusion de documents scientifiques de niveau recherche, publiés ou non, émanant des établissements d'enseignement et de recherche français ou étrangers, des laboratoires publics ou privés. 


\title{
INTERFACE RECOMBINATION IN GaAS-GaALAS QUANTUM WELLS
}

\author{
B. SERMAGE, M.F. PEREIRA Jr, F. ALEXANDRE, J . BEERENS, \\ R. AZOULAY, C. TALLOT, A.M. JEAN-LOUIS and D. MEICHENIN
}

Centre National d'Etudes des Télécommunications, 196. Avenue Henrt Ravera, F-92220 Bagneux, France

\begin{abstract}
Résumé : La durée de vie non radiative dans des doubles hétérostructures GaAs-GaAlAs non dopées fabriqués en EJM et EOM a été étudiée en utilisant une méthode de déclin de luminescence avec une résolution de $10 \mathrm{ps}$. La vitesse de recombinaison à l'interface $S$ est obtenue en étudiant des séries d'échantilions ayant différentes épaisseurs d de GaAs lentre $1 \mu \mathrm{m}$ et $20 \mathrm{~A})$. Pour chaque série, $S$ est constant quand $d$ est supérieur à $200 \AA$. Quand $d$ est inférieur à 200 A, S croit du fait de $\mathrm{I}$ 'augmentation de la probabilité de présence des porteurs dans la barrière quand l'épaisseur du puits décroit.

Abstract : Non radiative carriers lifetime has been studied in MBE and MOCVD grown GaAs-GaAlAs undoped double heterostructures by luminescence decay technique. Interface recombination velocity is obtained by studying series of samples with different GaAs layer thicknesses d (between $1 \mu \mathrm{m}$ and $20 \mathrm{~A})$. For each series $S$ is constant when $d$ is larger than $200 \AA$. When $d$ is smaller than $200 \AA$, $S$ increases due to the increase of the leaking of the carriers wave functions in the barrier when the well thickness decreases.
\end{abstract}

I - INTRODUCTION : The threshold current in double heterostructure lasers is proportional to the recombination probability in the active layer $1 / \tau$. In the case of the GaAs-GaAlAs lasers, the recombination probability has two components : the radiative one $1 / \tau_{r}$ and the non radiative one $1 / \tau_{n r}$.

$$
1 / \tau=1 / \tau_{r}+1 / \tau_{n r}
$$

The laser will be of practical use if $\tau_{n r}$ is large compared to the radiative life time at threshold carrier density $(\sim 4 \mathrm{~ns})$. The non radiative recombination probability, $1 / \tau_{n r}$ (called Shockley-Read) is the sum of the bulk and the interface recombination probabilities :

$$
1 / \tau_{n r}=1 / \tau_{n r b}+S / d
$$

where $d$ is the GaAs layer thickness and $S=S_{1}+S_{2}$ is the sum of the interface recombination velocity at the two interfaces. Eq. $2^{2}$ is valid when the GaAs layer thickness $d$ is small compared to the carrier diffusion length which is the case here.

Quantum well lasers have proved to be interesting devices (1) (smaller threshold current, larger modulation frequency). However in such small active layer thicknesses $(d \sim 50-100 \AA)$, interface recombination can be dramatic. In ref. 2, it was observed that interface recombination was smaller in quantum wells than in large GaAs layers, contrarily to what was expected theoretically due to an increase of the leaking of the carriers wave functions in the barriers when the thickness of the GaAs layer decreases (3). In fact, luminescence decay observed in ref. 2 was that of the GaAs buffer. Indeed in ref. 2, the $0.514 \mu \mathrm{m}$ excitation laser, excited both the AlGaAs barriers, the quantum well and the GaAs buffer and the only spectral selection before the detector was a low pass filter which cut the luminescence of the AlGaAs barriers and the $0.514 \mu \mathrm{m}$ laser. Careful measurements done here with a streak camera and a monochromator show that the inter- 
face recombination velocity increases in quantum wells as expected.

II - EXPERIMENT : We have studied two series of MBE grown samples and one series of MOCVO grown samples. The samples were undoped. The first MBE series was grown in July 84 and was the same as the one studied in ref. 2 and the second MBE series was grown in Dec. 86. In all the cases, the samples consisted of a GaAs layer of thickness $d$ included between two Ga $1-x^{A 1} x^{A s}$ confinement layers with $x$ approximatively equal to 0.3 . The thicknesses of the two confinement layers are $0.2 \mu \mathrm{m}$ and $0.1 \mu \mathrm{m}$ in the first MBE series, $0.5 \mu \mathrm{m}$ and $1 \mu \mathrm{m}$ in the second MBE series, $0.5 \mu \mathrm{m}$ and $1 \mu \mathrm{m}$ in the MOCVD series. We have observed independently that the thickness of the first confinement layer has a small influence on $S$ (about a factor of 2 between $200 \&$ and $0.5 \mathrm{\mu m}$ ) which cannot explain the large difference of the interface recombination velocity we observe in the different series. The thickness $d$ of the GaAs layer is varied between $1 \mu \mathrm{m}$ and 20 \& which allows to study the interface recombination velocity for large thicknesses and how it is modified in quantum wells. To have more chance that the interface quality is the same for all the samples of a series, all the structures contain a single GaAs layer. We have not studied multiquantum wells for which the interface quality is known to be improved (2). The MBE structures were grown at $690^{\circ} \mathrm{C}$ and the GaAs buffer layer was grown at $600^{\circ} \mathrm{C}$. The temperature rise was done at the beginning of the first GaATAs layer in the case of the first MBE series and at the end of the GaAs buffer layer in the case of the second MBE series.

The carriers lifetime is measured by luminescence decay. The sample is excited by the 3 ps pulses of a synchronously pumped Rhodamine $6 G$ mode locked dye laser at $0.58 \mu \mathrm{m}$ and the luminescence is spectrally selected by a small $10 \mathrm{~cm}$ monochromator. The decay is observed using a Hamamatsu two dimension streak camera so that we can observe on the screen of the camera the luminescence intensity as a function of wavelength and time and we can easily select the wavelength range we want to integrate for the decay measurement.

Examples of luminescence decay curves at low excitation are given on Fig. 1. Because the luminescence intensity $I_{L}$ is not proportional to the carriers density $n$, the carriers lifetime $\tau$ is not equal to the luminescence decay time constant $\tau_{l}$. However due to the short pulses and the good temporal resolution (10 ps), the carriers do not recombine during the excitation pulse and the carriers density at the end of the excitation pulse is proportionnal to the excitation power $P_{e x}$. Then the carriers lifetime $\tau$ at the end of the excitation pulse is related to the luminescence decay time at the end of the excitation pulse by :

$$
\tau=\tau_{L} \partial \log \left(I_{L}(0)\right) / \partial \log (n)=\tau_{L} \partial \log \left(I_{L}(0)\right) / \partial \log \left(P_{e x}\right)
$$

where $I_{L}(0)$ is the luminescence intensity at the end of the excitation pulse and the correction factor $\operatorname{aLog}\left(I_{L}(0)\right) / \partial \log \left(P_{e x}\right)$ can vary between 1 and 2 . For each

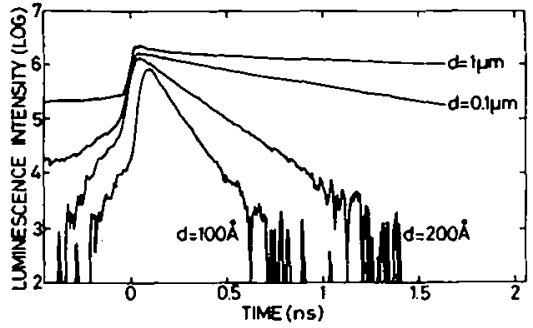

Fig. 1 : Examples of Tuminescence decay curves obtained with the streak camera at low excitation.

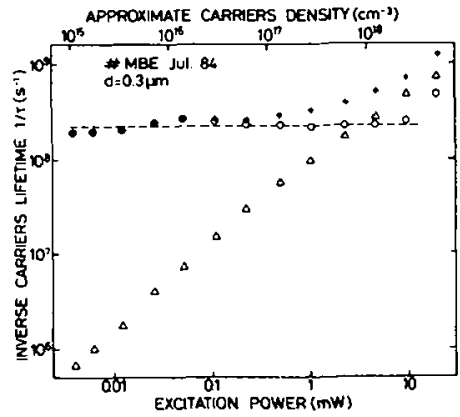

Fig. 2 : Variation with excitation of the total $(t)$ radiative $(\Delta)$ and non radiative (o) recombination probability. 
sample, we study the variation of $I_{L}(0)$ as a function of excitation power, calcu1ate the logarithmic derivative $a \log \left(I_{L}(0)\right) / \partial \log \left(P_{e x}\right)$ and measure if. The carriers life time $\tau$ is obtained using eq. 3 . On the other hand, we cafculate the ratio $I_{L}(0) / P_{e x}$ which is proportional to $1 / \tau_{r}$.

$$
I_{L}(0) \sim\left(\frac{\partial n}{\partial t}\right)_{r a d} \sim n \cdot \frac{1}{\tau_{r}} \sim P_{\text {ex }} \cdot \frac{1}{\tau_{r}}
$$

For each sample, we plot the recombination probability $1 / \tau$ and $I_{L} / P_{\text {ex }}$ as a function of the excitation power as shown in Fig. 2. The important point is that at low excitation, $1 / \tau$ is nearly constant while $1 / \tau_{r}$ decreases, which shows that this constant value is the probability of non radiative recombination $1 / \tau_{n r}$. Supposing that $1 / \tau_{n r}$ is constant which is nearly exact at low excitation, we can adjust the absolute value of $1 / \tau_{r}$ so that $1 / \tau_{n r}$ is constant as we have done on Fig. 2. Also shown on Fig. 2 is the approximate carriers density obtained by the measurement of the size of the laser beam on the sample and supposing that each photon creates an electron-hole pair in the active layer. This carriers density is certainly over-estimated because part of the carriers are created in the AlGaAs confinement layers from where they diffuse to the GaAs layer and part of these carriers are lost by recombination in the AlGaAs layer or at the free surface of the AlGaAs layer.

Fig. 3 gives the $1 / \tau$ curves as a function of the approximate carriers density for the samples of the first MBE series. The carriers density is estimated for the thick samples as previously and for the thin GaAs layers, we adjust by continuity of the radiative probability. For each sample, the constant value at low excitation gives the non radiative recombination probability. For the thick GaAs layers, the substrate luminescence is weak and does not modify the results. For the thin GaAs layers $(<100 \AA)$ the monochromator select the quantum well luminescence. Otherwise we would obtain the substrate non radiative recombination probability which is $7,10^{9} \mathrm{~s}^{-1}$.

On Fig. 4, we have plotted the $1 / \tau_{n r}$ value as a function of $1 / d$ for large thicknesses $(>200 \mathrm{~A})$. The experimental results follow eq. 3 with $S$ constant for each series. The large difference between the first and the second MBE series can be due either to the fact the shutter of the aluminium source has been changed between the two series or to the temperature rise time during the growth of the structure.

For small GaAs thicknesses, $(d<200$ A) $S$ increases as foreseen by Duggan et al.(3) due to the fact that the leaking of the electrons and holes wave functions

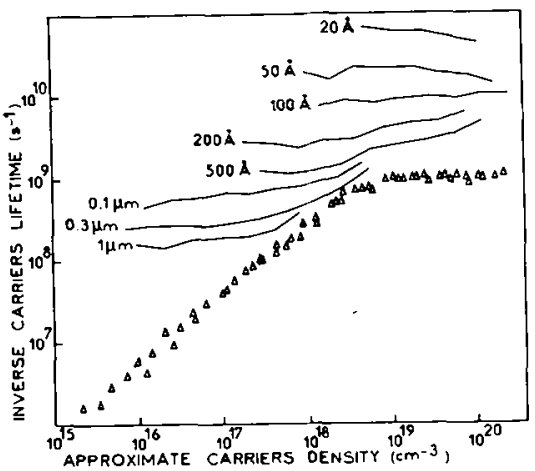

Fig. 3 : Variation with excitation of the total $(-)$ and radiative $(\Delta)$ recombination probability for different GaAs thicknesses.

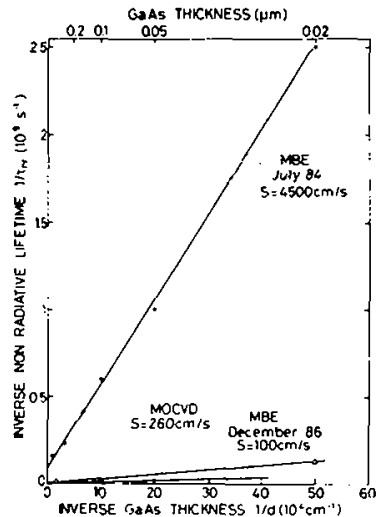

Fig. 4 : Variation of the non radiative recombination probability with the inverse GaAs layer thicknesses for the three series. 


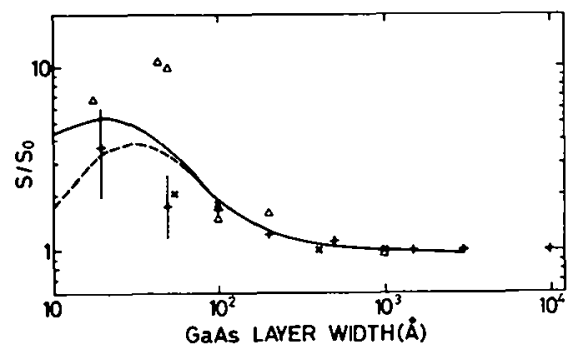

Fig. 5 : Ratio of the interface recombination velocity in GaAs quantum wells to its value in thick Tayers for the three series : first MBE series (+), second MBE series $(\Delta)$ and MOCVD series $(x)$. Calculated models for barrier

$(-;)$ and interface $(---)$ recombinations.

in the GaAlAs confinement layers increases. Fig. 5 shows the variation of $S / S$ for the small values of GaAs thicknesses where $S$ is the constant value for large GaAs thicknesses. For the three series, $S / S$ increases but the increase is not the same for the three series and we think that this is due to different sharpnesses of the interfaces. The large increase observed for the 50 \& width well of the second MBE series can be due to a resonance between the quantum well level and the levels of the recombination centers in the barrier. Also shown on Fig. 5 are the theoretical models of Duggan et al. calculated for an offset of the conduction band equal to $67 \%$ of the bandgap difference (4) and an Aluminium composition in the barrier equal to $30 \%$. The first model supposes that there is a constant density of recombination centers in the GaAlAs barrier and calculates the probability of presence of the electrons in the barriers. The second model supposes that all the recombinations happen at the exact interface and calculates the probability of presence of the electrons at the interface. He suppose this calculation is still valid for large GaAs layers where it gives a constant value $S_{0}$.

This increase of the interface recombination velocity is important for quantum well lasers. For example in the case of the second MBE series, the non radiative lifetime for $0.1 \mu \mathrm{m}$ thick layers is $100 \mathrm{~ns}$ which is negligible compared to the radiative lifetime in usual $D H$ lasers which is about 4 ns. However in the case of a quantum well of 50 \&, the non radiative lifetime is about 2 ns and increases the calculated threshold current from $110 \mathrm{~A} / \mathrm{cm}^{2}$ to $165 \mathrm{~A} / \mathrm{cm}^{2}$.

\section{REFERENCES}

1) S.D. Hersee, B. de Crëmoux and J.P. Duchemin, Appl. Phys. Lett. 44, 476 (1984)

2) B. Sermage, F. Alexandre, J.L. Liēvin, R. Azoulay, M. El Kaim, H. Le Person and J.Y. Marzin, Inst. Phys. Conf. Ser. $n^{\circ} 74,345$ (1985) and C. Khan Malek, B. Sermage, M.F. Pereira, M. El Kaim, F. Alexandre and R. Azoulay, Advanced materials for telecommunications 1986, Les éditions de Physique, p.239 (1986)

3) G. Duggan, H.J. Ralph and R.J. Elliott, Solid State Comm, 56, 17 (1985)

4) G. Danan, B. Etienne, F. Mollot, R. Planel, A.M. Jean-Louis, F. Alexandre, B. Jusserand, G. Le Roux, J.Y. Marzin, H. Savary and B. Sermage, Phys. Rev. $B, 35,6207$ (1987) 